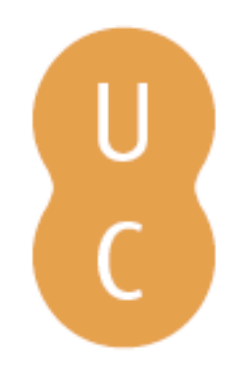

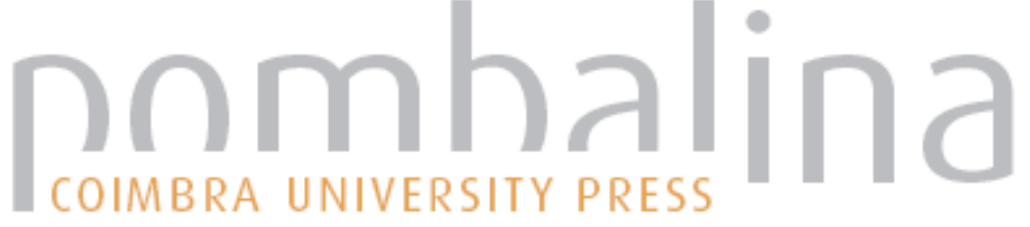

\section{Über Gewissheit und Selbstbezüglichkeit in Kants Vernunftglauben}

Autor(es): $\quad$ Nonnenmacher, Burkhard

Publicado por: Imprensa da Universidade de Coimbra

URL

persistente:

URI:http://hdl.handle.net/10316.2/31650

DOI:

DOI:http://dx.doi.org/10.14195/978-989-26-0205-9_15

Accessed : $\quad$ 26-Apr-2023 13:41:03

A navegação consulta e descarregamento dos títulos inseridos nas Bibliotecas Digitais UC Digitalis, UC Pombalina e UC Impactum, pressupõem a aceitação plena e sem reservas dos Termos e Condições de Uso destas Bibliotecas Digitais, disponíveis em https://digitalis.uc.pt/pt-pt/termos.

Conforme exposto nos referidos Termos e Condições de Uso, o descarregamento de títulos de acesso restrito requer uma licença válida de autorização devendo o utilizador aceder ao(s) documento(s) a partir de um endereço de IP da instituição detentora da supramencionada licença.

Ao utilizador é apenas permitido o descarregamento para uso pessoal, pelo que o emprego do(s) título(s) descarregado(s) para outro fim, designadamente comercial, carece de autorização do respetivo autor ou editor da obra.

Na medida em que todas as obras da UC Digitalis se encontram protegidas pelo Código do Direito de Autor e Direitos Conexos e demais legislação aplicável, toda a cópia, parcial ou total, deste documento, nos casos em que é legalmente admitida, deverá conter ou fazer-se acompanhar por este aviso. 
Edmundo Balsemão Pires

Burkhard Nonnenmacher

Stefan Büttner-von Stülpnagel

Editors

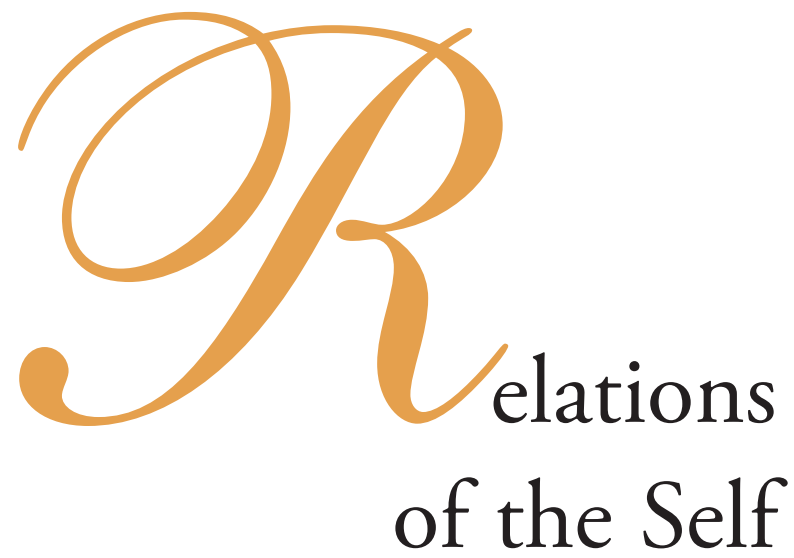




\section{Burkhard Nonnenmacher \\ München}

\section{ÜBER GEWISSHEIT UND SELBSTBEZÜGLICHKEIT IN KANTS VERNUNFTGLAUBEN}

Die folgenden Überlegungen beschäftigen sich mit dem in Kants Postulatenlehre entwickelten Begriff einer sogenannten „moralischen Gewissheit“1 über die Gültigkeit bestimmter theoretischer Sätze. - Mit einem kurzen Überblick über einige in Kants Postulatenlehre enthaltene und innerhalb der Kant-Forschung diskutierte Probleme wird in ihrem ersten Teil auf den Sachverhalt fokussiert, dass Kants Postulatenlehre eine unter dem Leitfaden praktischen Interesses erfolgende Setzung theoretischer Sätze nicht nur namhaft machen will, sondern auch für sich in Anspruch nimmt, dieses Setzen selbst als ein klar bestimmtes Verhältnis von praktischem und theoretischem Vernunftgebrauch begreifen zu können. - Der zweite Teil der Überlegungen möchte dann der Frage nachgehen, was für ein Verhältnis zwischen theoretischem und praktischem Vernunftgebrauch Kants Vernunftglauben konkret zu entwickeln beansprucht und dabei die These verfolgen, dass Kants „moralische Gewissheit“ als Selbstbezüglichkeitsstruktur zu interpretieren ist. - Im dritten und letzten Teil der Überlegungen soll zudem versucht werden, die zuvor zu Tage geförderte, innerhalb der Postulatenlehre virulente Selbstbezüglichkeitsstruktur näher zu bestimmen und kritisch zu reflektieren. Dabei werden auch einige in Kants Postulatenlehre enthaltene dialektische Probleme thematisiert. Ziel der Überlegungen wird es angesichts dieser Probleme vor allem sein, sich mit der Frage auseinanderzusetzen, mit welcher Methodologie Kant diese Schwierigkeiten zu lösen versucht und was das wiederum über den zuvor thematisierten, im Begriff der sogenannten „moralischen Gewißheit“ über die Gültigkeit bestimmter theoretischer Sätze enthaltenen Selbstbezüglichkeitsbegriff aussagt. Gewicht gelegt werden wird dabei nicht zuletzt auch auf die Frage, inwiefern in Kants Postulatenlehre nicht nur ein besonderes Lehrstück seiner praktischen Philosophie, sondern darüber hinaus auch ein die Architektonik des gesamten Kantischen Systems reflektierendes Lehrstück gesehen werden kann, das in besonderer Weise dazu geeignet ist, methodologische Differenzen zwischen Kants System und nachfolgenden Systemen der klassischen deutschen Philosophie offen zu legen.

\footnotetext{
${ }^{1}$ Kants Werke werden im Folgenden mit Band- und Seitenangabe nach der Akademie-Ausgabe (AA) zitiert (Immanuel Kant, Gesammelte Schriften, hrsg. von der Königlich Preußischen Akademie der Wissenschaften, Berlin ${ }^{2} 1910 \mathrm{ff}$.). Lediglich die Kritik der reinen Vernunft wird wie üblich nach der Paginierung der A- und B-Ausgabe zitiert. Vgl. AA Bd. III, S. B 857.
} 


\subsection{Probleme in Kants Postulatenlehre}

1. Kants Postulatenlehre nimmt ihren Ausgang vom Begriff des höchsten Guts. Dieser findet sich zu Beginn der Dialektik der Kritik der praktischen Vernunft darin bestimmt, „[d]aß Tugend (als die Würdigkeit glücklich zu sein)“ gemäß der Analytik der zweiten Kritik, zwar „die oberste Bedingung“" von allem uns Wünschenswerten und somit „das oberste Gut“ sein muss. Nicht soll Tugend damit aber bereits „das ganze und vollendete Gut, als Gegenstand des Begehrungsvermögens vernünftiger endlicher Wesen“ sein. Vielmehr soll so die zweite Kritik weiter - allererst "Glückseligkeit, ganz genau in Proportion der Sittlichkeit (als Werth der Person und deren Würdigkeit glücklich zu sein) ausgeteilt" das „höchste Gut“ einer Welt sein, in der weder Glückseligkeit auf Tugend noch umgekehrt Tugend auf Glückseligkeit reduziert werden kann. ${ }^{2}$ - Hiervon ausgehend beansprucht Kants Postulatenlehre darzulegen, dass es a) Voraussetzungen gibt, unter denen wir uns allein das höchste Gut als „Zusammenstimmung des Reichs der Natur mit dem Reiche der Sitten“3 denken können, und dass b) das Erfülltsein dieser Voraussetzungen in der Form theoretischer Sätze zu setzen „unabtrennlich zum praktischen Interesse der reinen Vernunft “4 gehört. - Ein „Postulat der reinen praktischen Vernunft“ nennt Kant vor diesem Hintergrund „einen theoretischen, als solchen aber nicht erweislichen Satz [...], sofern er einem a priori unbedingt geltenden praktischen Gesetze unzertrennlich anhängt" .

2. Bereits die schlichte Frage, wie diese, in Kants allgemeiner Definition eines Postulats genannte „unzertrennliche Anhänglichkeit“ zu denken ist, führt nun auf Schwierigkeiten. Denn fragt man erstens, weshalb ein theoretischer Satz einem a priori gültigen praktischen Gesetze unzertrennlich anhängen soll, und fragt man zweitens, was der Fall ist, wenn ein theoretischer Satz einem a priori gültigen praktischen Gesetze unzertrennlich anhängt, dann zeigt sich, dass Kants Antworten auf diese zum einen auf die moralische Motivation und zum anderen auf die theoretisch-systemische Deskription des Postulierens abzielenden Fragen in der Tat einigen Diskussionsbedarf hinsichtlich anderer zentraler Ergebnisse der Vernunftkritik mit sich bringen. Ein wichtiger Grund dafür ist, dass Kant den Gehalt seiner Postulatenlehre darüber expliziert, dass - so Kant - qua praktischem Postulieren gerade keine mit der Dialektik der Kritik der reinen Vernunft in Widerspruch stehende theoretische Existenz-Beweise geführt werden sollen ${ }^{6}$ und gleichwohl den Postulaten aber auch nicht nur

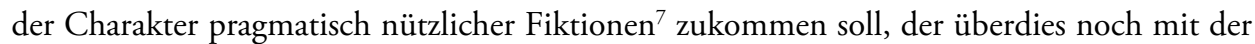
Analytik der Kritik der praktischen Vernunft in Widerspruch stehen könnte ${ }^{8}$. Vielmehr soll -

\footnotetext{
${ }^{2} \mathrm{Vgl}$. AA Bd. V, S. $110 \mathrm{ff}$.

${ }^{3}$ AA Bd. V, S. 145.

${ }^{4}$ AA Bd. V, S. 121.

${ }^{5}$ AA Bd. V, S. 122.

${ }^{6} \mathrm{Vgl}$. AA Bd. III, S. B 662 und AA Bd. V, S. $133 \mathrm{ff}$.

${ }^{7}$ Vgl. Otfried Höffe, Immanuel Kant, München 4. Aufl. 1996, S. 249.

${ }^{8}$ AA Bd. V, S. $125 f$.
} 
so Kant weiter - den Postulaten ein die theoretische Vernunft erweiternder Charakter zukommen ${ }^{9}$, der allein aus Praxis begründet wiederum aber nur soweit dem Postulierten eine praktische Realität ${ }^{10}$ zuspricht, dass hiermit die zentralen Ergebnisse der Dialektik der ersten Kritik unversehrt bleiben können ${ }^{11}$.

3. Eine kritische, ihren Sinn und Gehalt allererst in den Blick bringende Auseinandersetzung mit Kants Postulatenlehre hat ihren Fokus vor diesem Hintergrund vor allem auf zwei Probleme zu richten: Zum einen ist dies die Frage, inwiefern Kants Postulatenlehre in Rücksicht auf die Analytik der Kritik der praktischen Vernunft dazu in der Lage ist, die moralisch-praktische Notwendigkeit des Postulierens ${ }^{12}$ so auseinanderzusetzen, dass dabei deutlich wird, inwiefern es nach Kant gerade nicht zur, sondern aus Sittlichkeit notwendig sein soll, die Unsterblichkeit der Seele und das Dasein Gottes zu postulieren ${ }^{13}$. Zum anderen ist dies die Frage, inwiefern Kants Postulatenlehre mit Blick auf die Dialektik der Kritik der reinen Vernunft dazu in der Lage ist, das „Fürwahrhalten aus einem Bedürfnisse der reinen Vernunft "14 selbst zu bestimmen, sodass nachvollziehbar wird, was es heißen soll, „daß ich moralisch gewiß bin, daß ein Gott sei“"15, ohne dass damit behauptet ist, dass „dem [theoretisch] Zweifelgläubigen bewiesen [wird], daß ein Gott sei“16.

4. Kant nimmt nun für sich in Anspruch, in der Tat eine Antwort auf beide Fragen gegeben zu haben und zwar dies in der Entwicklung seines von ihm sogenannten „reinen praktischen Vernunftglaubens". ${ }^{17}$ - Für eine kritische Auseinandersetzung mit Kants Postulatenlehre, bedeutet das zunächst einmal konzedieren zu müssen, dass diese ihre Beweise für die unzertrennliche Anhänglichkeit bestimmter theoretischer Sätze an ein a priori unbedingt geltendes praktisches Gesetz nicht unabhängig von einem diesen Beweisen nachgeordneten Glaubensbegriff führen zu können behauptet, indem es nach Kant eben gerade dieser Glaubensbegriff erlauben soll, eine praktisch motivierte Artikulation bestimmter theoretischer Sätze so fassbar zu machen, dass hiermit in sinnvoller und klar umgrenzter Weise von praktischen Beweisen die Rede sein kann, ohne dass damit Inkonsistenzen mit dem übrigen kritischen Geschäft in Kauf genommen werden müssten. - Zweitens ist damit aber zugleich auch noch einmal unterstrichen, dass sich eine kritische Auseinandersetzung mit Kants Postulatenlehre keineswegs darauf beschränken kann, sich allein mit der Frage nach der praktischen Notwendigkeit der Postulatenlehre unter besonderer Ausrichtung auf Kants Begründung seines Begriffs des höchsten Guts zu beschäftigen. Vielmehr hat diese sich ebensosehr mit der Frage auseinanderzusetzen, welches explikative Instrumentarium der Kantische Begriff eines praktischen Vernunftglaubens dafür bereitstellt, eine unter dem Leitfaden

\footnotetext{
${ }^{9}$ AA Bd. V, S. 133.

${ }^{10}$ Vgl. Günter Zöller, Theoretische Gegenstandsbeziehung bei Kant, Berlin 1984, S. 285.

${ }^{11} \mathrm{Vgl}$. AA Bd. V, S. 134-136.

${ }^{12} \mathrm{Vgl}$. AA Bd. V, S. 125.

${ }^{13}$ Vgl. AA Bd. V, S. 451 Anm., sowie Bd. VI, S. 5.

${ }^{14} \mathrm{Vgl}$. AA Bd. V, S. $142 \mathrm{ff}$.

${ }^{15} \mathrm{Vgl}$. AA Bd. III, S. B 857.

${ }^{16}$ AA Bd. V, S. 450f. Anm.

${ }^{17}$ Vgl. AA Bd. V, S. 146.
} 
praktischen Interesses erfolgende Setzung theoretischer Sätze ${ }^{18}$ als ein klar bestimmtes Verhältnis von praktischem und theoretischem Vernunftgebrauch begreifen zu können ${ }^{19}$, denn allererst eine zusätzlich zur Reflexion der praktischen Notwendigkeit des Postulierens geführte Analyse der Kantschen Bestimmung dieses Verhältnisses kann deutlich machen, was es nach Kant heißen soll, dass ich moralisch gewiss bin, dass ein Gott ist ${ }^{20}$, weil hier die Frage danach, wie und an welchem Ort Kants Postulatenlehre ihrem eigenem Verständnis nach operiert oder operieren können soll, eben nicht nur dahingehend beantwortet ist, dass es aus und nicht zur Sittlichkeit notwendig ist, das Dasein Gottes zu setzen ${ }^{21}$, sondern dabei vielmehr auch das Verhältnis des theoretischen und des praktischen Moments des Postulierens reflektiert und bestimmt in den Blick gebracht wird ${ }^{22}$.

Ganz in diesem Sinne möchte ich mich im Folgenden der Frage zuwenden, was für ein Verhältnis zwischen theoretischem und praktischem Vernunftgebrauch Kants Vernunftglauben eigentlich konkret zu entwickeln beansprucht und dabei die These verfolgen, dass Kants innerhalb seiner Lehre eines praktischen Vernunftglaubens benannte „moralische Gewissheit“ als Selbstbezüglichkeitsstruktur zu interpretieren ist.

1.2 „Ein Zweck, welchen sich zu machen, schon sittliche Grundsätze voraussetzt. “23

1. Bevor ich fortfahren kann, ist zunächst eine kurze Bemerkung zu machen zum Verhältnis zwischen der Frage nach der moralisch-praktischen Notwendigkeit des Postulierens auf der einen Seite und der Frage nach der Bestimmung des Verhältnisses zwischen theoretischem und praktischem Vernunftgebrauch in Kants praktischem Vernunftglauben auf der anderen Seite.

Unmittelbar mag es scheinen, dass jede der beiden Fragen auf einen anderen Gegenstand gerichtet ist. Denn zielt die erste auf die Reflexion der praktischen Gründe für die gesetzte Möglichkeit des höchsten Guts und auf die Kohärenzfähigkeit dieser Gründe mit der Analytik der Kritik der praktischen Vernunft, so zielt die zweite Frage mit Blick auf Kants Lehre vom Primat des Praktischen ${ }^{24}$ auf die Frage, inwiefern Kants Postulatenlehre vor dem Hintergrund der Dialektik der Kritik der reinen Vernunft dazu in der Lage ist, den theoretischen Status und die praktische Realität des Postulierens selbst zu bestimmen. - So richtig dies ist, so sehr gilt aber zugleich, dass die zweite Frage keineswegs isoliert von der ersten Frage zu behandeln ist, weil die Art der Beantwortung der Frage nach der praktischen Notwendigkeit des Postulierens bereits wichtige Vorentscheidungen für die Beantwortung der zweiten Frage trifft. - Deshalb werde ich jetzt auch kurz auf diese praktische Frage eingehen.

\footnotetext{
${ }^{18} \mathrm{Vgl}$. AA Bd. III, S. B 833.

${ }^{19}$ Vgl. Michael Albrecht, Kants Antinomie der praktischen Vernunft, Hildesheim 1978, S. $167 \mathrm{ff}$.

${ }^{20} \mathrm{Vgl}$. AA Bd. III, S. B 857.

${ }^{21} \mathrm{Vgl}$. AA Bd. V, S. 451 Anm.

${ }^{22} \mathrm{Vgl}$. insbes. AA Bd. V, SS. 132-148.

${ }^{23} \mathrm{Vgl}$. AA Bd. VI, S. 5.

${ }^{24} \mathrm{Vgl}$. AA Bd. V, S. $119 \mathrm{ff}$.
} 
Im Unterschied zu sich ausführlicher mit dem Begriff der praktischen Motivation in Kants Postulatenlehre auseinandersetzenden und diesbezüglich unterschiedliche Lösungsstrategien diskutierenden Untersuchungen ${ }^{25}$, werde ich mich hier aber damit begnügen, nur einen kleinen Teilaspekt einer möglichen Antwortstrategie auf diese Frage zu skizzieren, um hiervon ausgehend dann meine eigentliche Fragestellung weiter zu entwickeln.

2. Wie oben bereits genannt, ergibt sich die wichtigste Konsistenzfrage, die im Hinblick auf die Analytik der Kritik der praktischen Vernunft an Kants Postulatenlehre zu richten ist, daraus, dass Kants zu Beginn von 1.1 zitierte Bestimmung des höchsten Guts im Sinne der Analytik der zweiten Kritik keinesfalls so verstanden werden darf, als wolle sie im Sinne einer sich für das Bedürfnis nach endlicher Glückseligkeit ${ }^{26}$ lohnenden Sittlichkeit zusätzliche Stütztriebfedern für eine Befolgung des Sittengesetzes ins Feld führen. - Will sie dies nicht, was Kant ab der zweiten Kritik mit Nachdruck betont ${ }^{27}$, stellt sich nämlich die Frage, wie sie dann zu verstehen ist, sodass klar wird, nicht nur dass, sondern warum es nach Kant nicht zur, sondern durch Sittlichkeit notwendig ist, die Unsterblichkeit der Seele und das Dasein Gottes zu setzen.

3. Um eine mögliche Strategie der Beantwortung dieser Frage zu skizzieren, möchte ich an dieser Stelle zunächst einmal kurz auf Kants Metaphysik der Sitten (1797) zu sprechen kommen, auch wenn diese den Versionen der Postulatenlehre der drei Kritiken (1781, 1788, 1790) und ihrer Rezeption in der Religionsschrift (1793) freilich erst später nachgefolgt ist.

In der Tugendlehre der Metaphysik der Sitten begegnet uns der Gedanke, dass gerade aus Sittlichkeit das einem jeden vernünftigen, aber endlichen Wesen aufgedrungene Problem seiner natürlichen Bedürftigkeit ${ }^{28}$ zu berücksichtigen ist. Der $\$ 10$ der Tugendlehre, überschrieben „Vom Geize“, argumentiert in diesem Sinne zum Beispiel folgendermaßen: Der karge Geiz verletzt die Pflicht gegenüber sich selbst, weil er „Verengung [...] der Mittel des Wohlleben[s] unter das Maß des wahren Bedürfnisses“29 ist. Denn dies bedeutet: „sklavische Unterwerfung seiner selbst unter die Glücksgüter, ihrer nicht Herr zu sein, welches Verletzung der Pflicht gegenüber sich selbst ist. Sie [diese Frugal-Unterwerfung, die nicht zu kontrollierende Affekte nach sich zieht, Vf.] ist der Liberalität (liberalitas moralis) der Denkungsart überhaupt [...], d.i. dem Prinzip der Unabhängigkeit von allem anderen, außer von dem Gesetz, entgegengesetzt, und Defraudation, die das Subject an sich selbst begeht. “30

Auf Kants Postulatenlehre von der Metaphysik der Sitten zurückblickend scheint mir dieser Gedanke deswegen interessant zu sein, weil sich hier deutlich zeigt, dass aus Sittlichkeit deren mögliche Negationen zu berücksichtigen gerade nicht damit gleichgesetzt werden kann, aus einem nicht-sittlichen Standpunkt heraus das Sittengesetz zu befolgen. Ganz in

${ }^{25}$ Vgl. Kaus Düsing, „Das Problem des höchsten Gutes in Kants praktischer Philosophie“, in: Kant-Studien Bd. 62, SS. 5-42, sowie Michael Albrecht, Kants Antinomie der praktischen Vernunft, Hildesheim 1978, S. 43ff.

${ }^{26} \mathrm{Vgl}$. AA Bd. V, S. 25.

${ }^{27} \mathrm{Vgl}$. in der Kritik der praktischen Vernunft AA Bd. V, S. 125f., in der Kritik der Urteilskraft AA Bd. V, S. 451 Anm. sowie in Die Religion innerhalb der Grenzen der bloßen Vernunft AA Bd. VI, S. 5.

${ }^{28} \mathrm{Vgl}$. AA Bd. V, S. 25.

${ }^{29}$ AA Bd. VI, S. 432.

${ }^{30}$ AA Bd. VI, S. 434. 
diesem Sinne geht es im zitierten Paragraphen der Tugendlehre nämlich keinesfalls darum, heteronome Stütztriebfedern für eine Befolgung des Sittengesetzes ins Feld zu führen, sondern vielmehr geht es allein darum, aus Pflicht die Blockade der formalen Bestimmung des Willens durch materiale, endliche Bestimmungsgründe eben dadurch auszuräumen, dass die Grenzen bestimmt werden, innerhalb denen diesen materialen, sinnlichen Affektionen unserer Willkür sogar nachgegeben werden muss.

4. $\mathrm{Zu}$ fragen bleibt hiermit dann freilich, ob und gegebenenfalls wie eine sich technischpraktischer $^{31}$ Überlegungen bedienende Praxis im Näheren zu denken ist und inwieweit zweitens im Zuge solcher Überlegungen gerade die Befriedigung endlicher Bedürfnisse zur Verwirklichung des Sittengesetzes selbst werden kann. - Ganz gleich jedoch, wie schwierig diese unmittelbar an Kants Metaphysik der Sitten zu stellenden Fragen für sich genommen bereits sind, mir scheint, auch Kants Postulatenlehre kann es nicht umgehen, sich mit solchen Fragen auseinandersetzen. Denn in der Tat lässt sich das Muster einer sich im Dienste ihrer selbst ihres Andern bedienenden Praxis auch bereits innerhalb der Postulatenlehre erkennen.

Verwiesen sei hier nur auf die berühmte, auf Spinoza zielende Schlussbemerkung des $\$$ 87 der Kritik der Urteilskraft, wo es heißt, dass bei einem rechtschaffenen Manne, der sich dennoch moralisch verpflichtet fühlt, auch wenn er von der Realisierbarkeit des höchsten Guts theoretisch nicht überzeugt ist, eben diese theoretische Unsicherheit nicht ohne eine Schwächung gegenüber dem Gefühl der Achtung fürs Gesetz und hiermit nicht „ohne einen der moralischen Gesinnung widerfahrenden Abbruch“ geschehen kann, weshalb eben daraus für jenen, der dies reflektiert und „dem Rufe seiner sittlichen inneren Bestimmung anhänglich bleiben“ will, gefolgert wird: „so muß er, welches er auch gar wohl thun kann, indem es an sich wenigstens nicht widersprechend ist, in praktischer Absicht, d.i. um sich wenigstens von der Möglichkeit des ihm moralisch vorgeschriebenen Endzwecks einen Begriff zu machen, das Dasein eines moralischen Welturhebers, d.i. Gottes, annehmen. “"32

5. Hinsichtlich der Frage nach der praktischen Notwendigkeit des Postulierens möchte ich in meinen folgenden Überlegungen zur Frage nach der Bestimmung des Verhältnisses von theoretischem und praktischem Vernunftgebrauch innerhalb der Postulatenlehre vor diesem Hintergrund und gemäß Kants eigenen Beteuerungen davon ausgehen, dass diese aus dem Sittengesetz selbst dessen Kontextualisierung für endliche Vernunftwesen abzuleiten versucht. Das heißt ich versuche Kants Postulatenlehre so zu begreifen, dass mit ihr nicht nur äußerlich die Gültigkeit des Sittengesetzes für endliche Wesen gesichert wird, indem von einem Standpunkt $\mathrm{C}$ aus zum Zwecke der Gültigkeit von A ein B instantiiert wird. Vielmehr verstehe ich Kants Postulatenlehre so, dass aus der unbedingten Gültigkeit des Sittengesetzes auch die Notwendigkeit der Reflexion über ihre Realisierungsbedingungen in endlichen Vernunftwesen gefolgert wird, was dann zum Postulieren weiterer gültiger Urteile im Dienste dieser ersten Gültigkeit führend aber gerade nicht vergessen machen kann, dass eben diese Reflexionen selbst als wie auch immer dann im Näheren zu bestimmender Fall dieser ersten Gültigkeit zu begreifen sind. - Hiermit ist aber auch bereits zur Frage nach der innerhalb von Kants Postulatenlehre ausgesprochenen Verhältnisbestimmung von theoretischem

\footnotetext{
${ }^{31}$ Zur Unterscheidung von „moralisch-praktisch“ und „technisch-praktisch“ vgl. AA Bd. V, S. 172.

${ }^{32}$ AA Bd. V, S. 452 f.
} 
und praktischem Vernunftgebrauch übergeleitet und ich komme damit zum zweiten Teil meiner Überlegungen.

2.

2.1 Die Frage nach dem Fürwahrhalten aus einem Bedürfnis der reinen Vernunft

1. Nicht zu übersehen ist, dass wo in der Tugendlehre von der „Verengung seines eigenen Genusses der Mittel zum Wohlleben unter das Maß des wahren eigenen Bedürfnisses “33 die Rede ist, da ist hingegen in Kants Postulatenlehre von der Sinnhaftigkeit- oder Sinnlosigkeit der gesamten Welt die Rede ${ }^{34}$, d.i. also von (endlichen) Totalitätsvorstellungen, womit klar ist, dass die Postulatenlehre die beschriebene Argumentationsstruktur der Metaphysik der Sitten von vornherein auf einer ganz anderen, theoretische Positionen betreffenden Ebene zu entfalten versucht. Nicht geht es der Postulatenlehre nämlich nur um die Frage, wie Praxis als sich anthropologischer Reflexionen bedienend sich in deren sittlichkeitsrelevanten Abschätzung Gebote und Verbote generieren kann, sondern vielmehr geht es ihr darum, bestimmte theoretische Unentschiedenheiten auf deren $\mathrm{Zu}$ - oder Abträglichkeit für die Realisierbarkeit von Sittlichkeit in endlichen Vernunftwesen zu bewerten ${ }^{35}$, um daraus dann ebenfalls aus Sittlichkeit Schlüsse zu ziehen, die dann angesichts ihres theoretische Aussagen evozierenden Charakters aber nicht Ge- oder Verbote, sondern Postulate genannt werden.

2. Während sich mit 1.2 also bereits eine mögliche Lösung dafür abzeichnet, wie Kants Postulatenlehre so verstanden werden kann, dass sie gerade nicht darum bemüht ist, zusätzliche, heteronome Stütztriebfedern für die Befolgung des Sittengesetzes einzuführen, indem in der Tat aus Sittlichkeit deren mögliche Negationen zu berücksichtigen gerade nicht damit gleichgesetzt werden kann, aus einem nicht-sittlichen Standpunkt heraus das Sittengesetz zu befolgen, fangen die rücksichtlich Kants theoretischer Philosophie an seine Postulatenlehre zu stellenden Konsistenzfragen damit allererst an. - Zu fragen ist nämlich, was dieses Evozieren theoretischer Vorstellungen im Dienste der Praxis mit Blick auf die Erkenntniskritik der Kritik der reinen Vernunft eigentlich sein soll? Denn mag der qua Tugendlehre propagierte Kultivierungsgedanke auch als eine sich technisch-praktischer Überlegungen bedienende Praxis auseinandergelegt werden können, stößt die Frage nach der genaueren Bestimmbarkeit dieses Verhältnisses innerhalb der Postulatenlehre ja auf das zusätzliche Problem, bestimmen zu müssen, wie denn theoretische Sätze praktisch motiviert in dem Wissen darum für wahr gehalten werden können sollen, dass die theoretische Unsicherheit über deren Wahrheitsgehalt unvermeidlich einen der moralischen Gesinnung widerfahrenden Abbruch nach sich zöge ${ }^{36}$.

${ }^{33}$ Vgl. AA Bd. VI, S. 432.

${ }^{34}$ Vgl. Reinhard Brandt, Die Bestimmung des Menschen bei Kant, Hamburg 2007, S. 371.

${ }^{35}$ Vgl. hierzu besonders auch das Kapitel „Von dem Interesse der Vernunft bei diesem ihrem Widerstreite“ der Kritik der reinen Vernunft, AA Bd. III, SS. B 490-504.

${ }^{36}$ Vgl. hierzu abermals Kants Spinoza-Anmerkung in der Kritik der Urteilskraft, AA Bd. V, S. $452 f$. 


\subsection{Selbstvergewisserung und Selbstrealisierung}

1. Eine wichtige, sich in diesem Zusammenhang stellende Frage besteht nun darin, ob solche im Dienste der Freiheit ersehenen „Wahrheiten“ nur dann ihren Dienst erfüllen können, wenn in ihrer theoretischen Artikulation ihre praktische Motivation latent bleibt, oder ob der mit ihnen intendierte Zweck auch im Wissen um diesen erfüllt werden kann. Gefragt werden muss also, ob eine moralisch-praktisch motivierte, sich technisch-praktischer Überlegungen bedienende Argumentation für eine bestimmte theoretische Weltsicht nur dergestalt zur Tat schreiten kann, dass sie gleichsam blitzartig Dogmen für die Theorie aussendet, die ihre Nützlichkeit gerade darin entfalten, dass ihre Nützlichkeit nicht gewusst wird, - oder ob dieser Akt des Eingreifens der Praxis in die Theorie auch bewusst vollzogen werden kann, was offensichtlich die von der Postulatenlehre in ihrer Auseinandersetzung des Begriffs eines praktischen Vernunftglaubens intendierte Variante ist.

2. Wie aber könnte dieser Eingriff der Praxis in die Theorie aussehen? - Im Folgenden möchte ich die These verfolgen, dass Kants Postulatenlehre diese Frage damit beantwortet, dass sich qua praktischem Glauben die Praxis eine Ordnung der Welt darin „er-sehen“ soll ${ }^{37}$, dass sich die Praxis im Sehen dieser Ordnung selbst sieht, eben weil sie dieses Sehen selbst ganz im Sinne der in 1.2 erwähnten Spinoza-Anmerkung des $\$ 87$ der Kritik der Urteilskraft - als ein bestimmtes Moment ihrer Selbstrealisation begreift. - Meine These ist also, dass sich Praxis qua moralischem Vernunftglauben in der Projektion einer ihr gemäßen Weltordnung ihrer selbst vergewissert, indem sie dieses Projizieren als Moment ihrer Selbstrealisation begreift. - Im Hinblick auf das zweite Postulat gesprochen wäre das Dasein Gottes damit genau darin gesetzt und "gewiss“, dass begriffen wird, dass dieses Setzen nichts als eine bestimmte Art von Selbstrealisation bedeutet, eben indem reflektiert wird, dass diese Sätze gesetzt werden müssen, um nicht die Anhänglichkeit an den Ruf der sittlichen inneren Bestimmung durch theoretische Unentschiedenheiten schwächen zu lassen ${ }^{38}$.

3. Geleitet zu sein scheint mir Kants Postulatenlehre somit von der Idee, dass die praktische Relevanz bestimmter theoretischer Sätze zu sehen und am Ort der Praxis selbst ${ }^{39}$ zu erwägen, gleichbedeutend damit sein soll, diese theoretischen Sätze als praktisch-wahre Sätze zu setzen und zu begreifen.

4. Unter genau dieser Idee, so meine These weiter, ließe sich dann auch näher bestimmen, was denn diese unzertrennliche Anhänglichkeit theoretischer, als solcher aber nicht erweislicher Sätze an das praktische Gesetz eigentlich ist, die Kants allgemeine Definition dessen, was er unter einem Postulat versteht, nennt. Denn diese unzertrennliche Anhänglichkeit bestünde im Sinne der genannten Leitidee offenbar darin, dass die Reflexion der Zweckmäßigkeit einer bestimmten Weltsicht für die Praxis vom Standpunkt des Zwecks aus, respektive im Interesse der Praxis gesehen, gerade als die Einnahme dieser Weltsicht selbst verstanden werden muss.

${ }^{37}$ Vgl. Allen W. Wood, Kant's Moral Religion, Ithaca 1970, S. $153 \mathrm{ff}$.

${ }^{38}$ Vgl. abermals AA Bd. V, S. 452.

${ }^{39}$ Den Gedanken und die Formulierung, dass sich die Postulatenlehre mit „am Ort der Praxis“ erwogenen theoretischen Aussagen beschäftigt, verdanke ich Peter Reisinger. Vgl. hierzu auch ders., Die logischen Voraussetzungen des Begriffs der Freiheit bei Kant und Hegel, Frankfurt a. M. 1967. 
5. Ihren Nukleus hätte die von Kant proklamierte unzertrennliche Anhänglichkeit bestimmter theoretischer Sätze an ein a priori unbedingt geltendes praktisches Gesetz damit aber offensichtlich in der die genannte Leitidee konkretisierenden Überzeugung, dass eine zunächst nur reflektierte praktische Relevanz einer bestimmten Weltsicht genau darin praktisch wirksam wird, dass diese Weltsicht selbst als Gegebenheit vorgestellt wird, d.i. gleichsam als theoretisch bejaht vorgestellt wird.

6. Gerade die Einnahme bestimmter theoretischer Vorstellungen würde qua Postulatenlehre damit also selbst zum ersten Vergewisserungspunkt und Begriff einer am Ort der Praxis begriffenen Zuträglichkeit bestimmter theoretischer Vorstellungen hinsichtlich der Realisierbarkeit der moralischen Gesinnung in endlichen Vernunftwesen.

Eben dieses schwierige Verhältnis von theoretischem und praktischem Vernunftgebrauch, so der erste Zielpunkt meiner Überlegungen, artikuliert und hat der praktische Vernunftglaube selbst zu begreifen. - Hiervon ausgehend möchte ich nun die skizzierte Struktur praktischen Glaubens bei Kant als Selbstverhältnis der Praxis näher bestimmen und dabei zeigen, dass Kants innerhalb seiner Postulatenlehre behauptete moralische Gewissheit über die Gültigkeit bestimmter theoretischer Sätze eine einen ganz bestimmten Begriff von Selbstbezüglichkeit thematisierende Struktur ist.

\subsection{Vernunftglaube und praktischer Selbstbezug}

1. Warum ist die moralische Gewissheit Kants nach den in 2.2 skizzierten Überlegungen als eine selbstbezügliche Struktur zu bezeichnen und vor allem was für eine selbstbezügliche Struktur benennt sie? - Ganz allgemein lässt sich diese Frage damit beantworten, dass mit Kants moralischer Gewissheit ein Sichrealisieren von Praxis in Theorie gedacht wird, das bedeutet, dass die Setzung von einem bestimmten theoretischen Satz in Einem a) als praktische Tat und b) als praktische Selbstvergewisserung verstanden werden muss. Denn Selbstbezüglichkeit ist damit dergestalt thematisiert, dass die Tat des Setzens bestimmter theoretischer Sätze als praktische Realisation mit der Reflexion dieser Tat zusammenfällt, nämlich im einfachen Setzen des theoretischen Satzes im Gewiss-sein um dessen Realisationsbedeutung für die Praxis.

2. Zusätzlich kann damit konstatiert werden, dass Kants moralische Gewissheit eine Struktur prinzipieller Selbstbezüglichkeit benennt, nämlich das Aufsichbezugnehmen eines Tuns, das von diesem Tun selbst gar nicht zu trennen ist.

3. Exakt in diesem Sinne wird m.E. auch erst die berühmte, dem Kanon-Kapitel der ersten Kritik entstammende Aussage Kants verständlich, die den Begriff des praktischen Glaubens als moralische Gewissheit mit dem Satz erläutert: „so muß ich nicht einmal sagen: es ist moralisch gewiß, daß ein Gott sei etc., sondern: ich bin moralisch gewiß etc. Das heißt: der Glaube an einen Gott und eine andere Welt ist mit meiner moralischen Gesinnung so verwebt, daß, so wenig ich Gefahr laufe, die letztere einzubüßen, eben so wenig besorge ich, daß mir der erste jemals entrissen werden könne. ${ }^{“ 40}$ Denn diese Verwebt- oder Verwobenheit

\footnotetext{
${ }^{40}$ AA Bd. III, S. B 857.
} 
besteht nach dem zuvor Gesagten eben genau darin, dass die assertorische Artikulation ${ }^{41}$ des theoretischen Satzes über die Existenz Gottes nichts als die praktische Tat ist, die a) das Setzen des theoretischen Satzes als ihre Realität setzt, b) in diesem Verständnis den einfachen theoretischen Satz setzt und c) damit in Einem dessen Inhalt und sich selbst als Praxis Wirklichkeit zu verleihen glaubt. ${ }^{42}$

Was für ein Begriff von Selbstbezüglichkeit ist damit aber mit Kants entwickelter Struktur einer moralischen Gewissheit über die Geltung bestimmter theoretischer, als solcher aber nicht erweislicher Sätze, artikuliert? Ich komme hiermit zum dritten Schritt meiner Überlegungen.

3.

\subsection{Gesetzter Glaube?}

1. Nach dem in 2.2 und 2.3 Entwickelten ist Praxis im praktischen Glauben nicht nur in irgendeiner Weise für sich, indem sie an etwas Bestimmtes glaubt, denn Praxis realisiert sich zugleich in diesem Glauben in einer bestimmten Weise selbst. Ja, es gilt sogar, eben dass sich Praxis qua Glauben in bestimmter Weise selbst realisiert, dies ist allererst der Grund dafür, dass Praxis im Glauben in bestimmter Weise für sich sein kann.

2. Bereits in 2.3 wurde deutlich, dass Kants praktischer Vernunftglaube damit ein Selbstverhältnis der Praxis namhaft zu machen scheint, in dem sich ein Ansichsein und dessen Fürsichsein nicht voneinander trennen lassen. Beides ist vielmehr eins aus der Perspektive des am Ort der Praxis selbst im Wissen um die praktische Zuträglichkeit gesetzten theoretischen Satzes.

Noch deutlicher wird dies jedoch, vergegenwärtigt man sich zudem, dass gerade das Wissen um diese Verwobenheit von An- und Fürsichsein der Praxis im praktischen Glaubensakt selbst Voraussetzung dafür zu sein scheint, dass ich moralisch gewiss sein kann über die Gültigkeit theoretischer Sätze, wie etwa, dass ein Gott ist. Denn nach dem zuvor Gesagten besteht diese moralische Gewissheit ja gerade darin, einen theoretischen Satz darin als wahr zu begreifen, dass eben dies zu tun als Realisierung eines praktischen Bedürfnisses begriffen wird und in diesem Sinne in Einem a) einem theoretischen Satz und b) einer bestimmten praktischen Selbstrealisierung Wirklichkeit verliehen wird und in diesem Sinne qua Glaubensakt ein Ansichsein, das zugleich ein Fürsichsein, und umgekehrt ein Fürsichsein, das zugleich ein Ansichsein ist, gesetzt und als praktisch wirklich geglaubt wird.

3. Was lässt sich damit festhalten? - Indem das Eine als das Andere gedacht wird - d.i. eine praktische Realität als Artikulation einer theoretischen Aussage und umgekehrt die Artikulation einer theoretischen Aussage als eine praktische Realität -, indem diese Identität gedacht wird, soll moralische Gewissheit möglich sein. - Realität hat die moralische Gewißheit

\footnotetext{
${ }^{41}$ Vgl. AA Bd. V, S. $4 f$.

${ }^{42}$ Ganz in diesem Sinne betont auch Hans Blumenberg, dass „die Eigenart der praktischen Gewißheit“ darin besteht, dass sie „weder Gewißheit „vor“ noch „nach“ einem Akt, sondern nur Gewissheit „in“ und „mit“ einem Akt sein [kann]“. Vgl. hierzu ders., „Kant und die Frage nach dem gnädigen Gott“, in: Studium Generale Bd. 7, S. 560.
} 
damit zunächst einmal darin, dass die Artikulation und Assertion eines theoretischen Satzes sich selbst als Glaubenswirklichkeit ausspricht, indem diese sich selbst als Einheit einer praktischen Selbstvergewisserung und Selbstrealisation begreift.

4. $\mathrm{Zu}$ fragen ist jedoch auch, ob die moralische Gewissheit im praktischen Glauben letztlich nur darin besteht, dass a) eine bestimmte theoretische Aussage als diese Einheit von An- und Fürsichsein der Praxis begriffen wird und b) eben dieses Begreifen im Setzen des einfachen theoretischen Satzes zugleich als die Realität dessen, die es ausspricht, gesetzt und geglaubt wird.

Dies würde nämlich bedeuten, dass auch die moralische Gewissheit des praktischen Vernunftglaubens letztlich nur so gut ist, wie diese Tat der Setzung dieser Identität von Anund Fürsichsein sich ihrer selbst sicher sein kann, d.i. ein Kriterium dafür hat, selbstreferentiell verwirklicht zu sein, wobei ich mit „selbstreferentiell verwirklicht zu sein“ hier meine, dass nicht nur gemäß den qua Kants Postulatenlehre entwickelten Verhältnissen ein performanter Akt allein theoretisch benannt wird, sondern dass dieser auch tatsächlich praktisch wirklich ist, wenn er als solcher gesetzt wird, womit er sozusagen sein Wahrheits-Kriterium an sich selbst haben müsste, etwa indem er gerade sein bloßes Gesetztsein als seine Wirklichkeit und An-und-fürsich-sein zu konstruieren begriffe ${ }^{43}$, um eben hierin - sich seiner selbst sicher ein praktisch wirkliches Bedürfnis zu sein und nicht nur eine Beschreibung desselben im Sinne einer bloß verstandenen, aber in ihrer willentlichen Verwirklichung nicht näher konkretisierten Logik von Kants Postulatenlehre.

5. Auf die Frage, inwieweit Kant dieses Gewissheits-Kriterium gerade nicht zur Verfügung stellen zu müssen meint, kann an dieser Stelle freilich nicht ausführlicher eingegangen werden. Deutlich gemacht werden soll hier nur, dass sich vor dem Hintergrund solcher Fragestellungen zeigt, dass Kants sogenannte moralische Gewissheit über die Gültigkeit bestimmter theoretischer Sätze eine Selbstbezüglichkeitsstruktur thematisiert, die selbst weitere Schwierigkeiten mit sich bringt, wenn man sie als praktisch wirkliche Struktur zu thematisieren und zu konkretisieren versucht. - Noch deutlicher wird dies im Folgenden.

\subsection{Die Frage nach der Trennbarkeit von Theorie und Praxis}

1. In 2.2 wurde herauszustellen versucht, dass qua Vernunftglauben die Praxis nicht etwas Anderes zu sich selbst sieht, sondern dass sie in ihrem Sehen eines theoretischen Sachverhalts sich selbst sieht. - Zusätzlich verkompliziert wird dieses Verhältnis nun jedoch durch den Umstand, dass die im Postulieren verfolgte Selbstrealisierung von Praxis nur dann gelingen kann, wenn die Praxis in ihrem Sehen eines theoretischen Sachverhalts gerade nicht nur sich selbst sieht, sondern vielmehr dieses tatsächlich als das Sehen eines theoretischen Sachverhalts begreift oder glaubt. Denn der Grund für die Statuierung bestimmter theoretischer Aussagen besteht mit 1.2 ja genau darin, dass damit der Reflexion der Abträglichkeit bestimmter theoretischer Unentschiedenheiten für die moralische Gesinnung

${ }^{43}$ Vgl. hierzu G.W.F. Hegel, Die Wissenschaft der Logik. Zweiter Band. Die subjective Logik oder Lehre vom Begriff, in: Georg Wilhelm Friedrich Hegel: Gesammelte Werke, hrsg. von der Rheinisch-Westfälischen Akademie der Wissenschaften, Hamburg 1968ff. (GW), Bd. 12, 12ff. 
Rechnung getragen werden soll. Dies wird aber nur dann der Fall sein, wenn die im Dienste der Praxis statuierten theoretischen Aussagen nicht nur als praktisch nützlich sein sollende Fiktionen erscheinen, sondern vielmehr praktisch wirksam werden können, indem sie nicht nur als gesetzte, sondern als tatsächliche Sachverhalte geglaubt werden dürfen.

2. Berücksichtigt man das, muss damit der von Kant behauptete „Zuwachs“, den „die theoretische Erkenntniß der reinen Vernunft “44 qua Postulatenlehre bekommen soll ${ }^{45}$, genauer unter die Lupe genommen werden. Denn für das zuletzt genannte, zusätzliche Problem scheinen sich unmittelbar nur zwei Lösungen anzubieten: Entweder man setzt wirklich diese Erkenntniserweiterung. Oder aber man konzediert schlicht, dass, auch wenn diese Erkenntniserweiterung selbst nur ein Postulat sein soll, gelten muss, dass der qua praktischem Glauben wirkliche Selbstbezug der Praxis nur dann praktisch wirksam wird, wenn der im Glauben wirkliche Selbstbezug sich selbst dergestalt sein Anderes ist, dass er sich selbst gerade nicht nur als praktischer, sondern als ein theoretische Ausmaße habender Akt begreift.

3. Beiden Lösungen hängt nun das Problem an, den Standpunkt rechtfertigen zu müssen, von dem aus sie beanspruchen, ihre Behauptungen machen zu können. Dabei ergibt sich folgendes Szenario: Versteht sich die erste Lösung als absoluter Standpunkt, so scheint dieser in seiner Genetik selbst nicht offengelegt werden zu können. Versteht sich die zweite, eine praktisch begründete Metaphysik verfolgende Lösung hingegen als nichts anderes als eine unter einem Primat des Praktischen durchexerzierte kritische Philosophie, dann ergibt sich hierbei aber auch ein Problem. Denn wenn im zweiten Fall die gesetzte Eigenständigkeit des theoretischen Inhalts allein im praktischen Interesse gründet, dann scheint dies in eine die Eigenständigkeit jeglicher Theorie ins Wanken bringende schlechtunendliche Dialektik zu führen, die unmittelbar keineswegs bereits als eine gelingende Artikulation der Aussage zu verstehen ist, dass „alles Interesse zuletzt praktisch ist, und selbst das der speculativen Vernunft nur bedingt und im praktischen Gebrauche allein vollständig ist " ${ }^{\text {" }}$.

4. $\mathrm{Zu}$ fragen ist vor diesem Hintergrund, ob Kants Lehre „Von dem Primat der reinen praktischen Vernunft in ihrer Verbindung mit der speculativen “47 innerhalb seiner Postulatenlehre überhaupt noch als ein nur bestimmte Probleme regeln sollendes Lehrstück erscheinen kann. Denn mit der zuletzt skizzierten Problematik geht es plötzlich ja nicht mehr nur um die Frage, ob praktische und theoretische Vernunft nur in einem bestimmten Widerstreit zwischen theoretischer Unentschiedenheit und praktischer Präferenz hinsichtlich der Gültigkeit bestimmter theoretischer Sätze a) einander koordiniert vorgestellt werden sollen, um damit einander widerstreitend zu bleiben, oder ob b) eben vielmehr im Sinne eines Primats des einen Vernunftgebrauchs über den anderen beide einander untergeordnet vorgestellt werden sollen. Nein, vielmehr scheinen sich nun in Berücksichtigung der praktischen Relevanz des „Zuwachs-Gedankens“ an die oben entwickelte Verwobenheit bestimmter theoretischer

\footnotetext{
${ }^{44}$ Vgl. AA Bd. V, S. 134.

${ }^{45}$ Das heißt der Zuwachs, „daß jene für sie sonst problematische (blos denkbare) Begriffe jetzt assertorisch für solche erklärt werden, denen wirklich Objecte zukommen, weil praktische Vernunft die Existenz derselben zur Möglichkeit ihres und zwar praktisch schlechthin nothwendigen Objects, des höchsten Guts, unvermeidlich bedarf, und die theoretische dadurch berechtigt wird, sie vorauszusetzen" (ebd.).

${ }^{46}$ AA Bd. V, S. 121.

${ }^{47}$ AA Bd. V, SS. 119-121.
} 
Aussagen mit unserer moralischen Gesinnung größere Probleme anzuschließen, die plötzlich eine prinzipielle Trennbarkeit von Theorie und Praxis in Frage stellen. - Im Folgenden möchte ich nun eine thetische Annäherung an die Kantische Lösung dieser Problematik zu unternehmen versuchen.

\subsection{Kants methodologisch reflektierte Trennung von Theorie und Praxis}

1. Prinzipiell scheint mir Kant seine Antwort auf die in 3.2 skizzierte Problematik darin zu geben, dass seine Lehre vom Primat des Praktischen keineswegs auf eine Unterwerfung sondern vielmehr nur auf eine Unterordnung ${ }^{48}$ des spekulativen unter den praktischen Vernunftgebrauch abzielt, die gerade nicht so zu verstehen ist, dass mit ihr die ganze eine Vernunft unmittelbar zu praktischer Vernunft wird, sondern die vielmehr „in Analogie zu

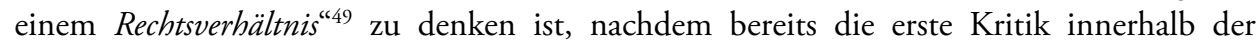
Transzendentalen Methodenlehre mit Nachdruck betont, dass die Vernunft „kein dictatorisches Ansehen hat“, sondern vielmehr jeder „Ausspruch [der sich selbst kritisierenden und ordnenden Vernunft, Vf.] jederzeit nichts als die Einstimmung freier Bürger ist, deren jeglicher seine Bedenklichkeiten, ja sogar sein veto ohne Zurückhalten muß äußern können" ${ }^{\text {"50 }}$.

Ganz in diesem Sinne scheint mir Kants Lehre vom Primat des Praktischen, wenn sie konkretisiert durch Kants Postulatenlehre a) eine Einheit von theoretischer und praktischer Vernunft in praktischer Rücksicht benennt, zugleich auch ebensosehr b) eine Trennung von theoretischem und praktischem Vernunftgebrauch in theoretischer Rücksicht darin betonen zu wollen, dass sie bei aller moralischen Notwendigkeit ${ }^{1}$ des praktischen Glaubens zugleich auch eine Freiwilligkeit ${ }^{2}$ desselben attestiert, indem sie ihn nicht zur Pflicht, sondern lediglich zu einem praktischen Bedürfnis ${ }^{3}$ erklärt.

2. Wie ist dieses Programm nun aber näher auszubuchstabieren, damit die in 3.2 an der Postulatenlehre vorstellig gewordene Verwobenheit von Theorie und Praxis ${ }^{54}$ gerade nicht zu einer völligen Aufhebung aller Differenz zwischen Theorie und Praxis führt? - Mein Versuch einer thetischen Annäherung an die Beantwortung dieser Frage lautet wie folgt:

3. Kants wiederholte Betonung davon, dass qua moralischem Glauben von der Realisierung eines objektiven Gebots unter subjektiven Bedingungen die Rede ist ${ }^{55}$, scheint unmittelbar betrachtet vor allem damit beschäftigt zu sein, im Sinne der Kritik des theoretischen Vernunftgebrauchs der Kritik der reinen Vernunft an einem Unterschied zwischen praktischem

\footnotetext{
${ }^{48} \mathrm{Vgl}$. AA Bd. V, S. 121.

${ }^{49} \mathrm{Vgl}$. Axel Hutter, Das Interesse der Vernunft, Hamburg 2003, S. $153 \mathrm{f}$.

${ }^{50} \mathrm{Vgl}$. AA Bd. III S. B 766. Vgl. hierzu abermals Axel Hutter, a.a.O.

${ }^{51} \mathrm{Vgl}$. AA Bd. V, S. 125.

${ }^{52} \mathrm{Vgl}$. AA Bd. V, S. 146.

${ }^{53} \mathrm{Vgl}$. AA Bd. V, S. 125.

${ }^{54} \mathrm{Vgl}$. AA Bd. III S. B 857.

${ }^{55} \mathrm{Vgl}$. AA Bd. V, S. $145 f$.
} 
und theoretischem Vernunftgebrauch dergestalt festzuhalten, dass qua Postulatenlehre eben nicht vergessen werden darf, dass nur in praktischer Absicht ventilierte theoretische Aussagen thematisiert werden.

In diesem Sinne lässt sich zunächst einmal folgender Unterschied festhalten, der durch die Formulierung eines unter subjektiven Bedingungen realisierten objektiven Gebots benannt wird: a) Das objektiv-Allgemeine, das nur unter einer subjektiven Bedingung wirklich gesetzt werden kann, erhebt diese subjektive Bedingung in den Stand der Allgemeingültigkeit selbst, wenn das objektiv Allgemeine an demjenigen Ort, an dem es nur unter subjektiven Bedingungen als wirklich gesetzt werden kann, als objektiv gültig und wirklich gesetzt wird. b) Eben diesen, die objektive Allgemeingültigkeit mit ihrer subjektiv-besonderen Bedingung zusammenschließenden Blickwinkel nimmt die Praxis als praktischer Vernunftglaube ein und „beweist“ in diesem Sinne die praktische Gültigkeit bestimmter theoretischer Sätze. c) Entgegen dieser in praktischer Rücksicht betonten Realität subjektiver Bedingungen des Setzens der Wirklichkeit von objektiv Gebotenem kann ein theoretisch teilnahmsloser Blick auf die Dinge hingegen zwischen objektiv allgemein Gebotenem einerseits und seiner subjektiv-besonderen Realisierungsbedingung andererseits unterscheiden. Eben damit ist für ihn das Allgemeine aber nicht schlechthin mit seiner besonderen Realisierung verknüpft, sondern nur von einem bestimmten Standpunkt aus und ebenso denkt Kants Lehre vom Primat des Praktischen qua Postulatenlehre zum Einen eine bestimmte, praktische Einheit von Theorie und Praxis und zum Anderen eine bestimmte, theoretische Differenz.

4. Unmittelbar ist das in 3.2 entwickelte dialektische Problem damit aber freilich noch immer nicht gelöst. Denn in dem im Interesse der Praxis stehenden „Zuwachs-Gedanken“ eine mögliche schlechtunendliche Dialektik erkennend beschäftigt dieses Problem ja nicht nur die Frage, nach einer Unterscheidbarkeit zwischen einer theoretischen Trennung und einer praktischen Einheit von Theorie und Praxis im zuletzt geschilderten Sinne, sondern vielmehr beschäftigt sich 3.2 bereits zusätzlich mit der Frage, was denn der Fall ist, wenn angesichts einer im Interesse der Praxis zu setzenden Erkenntniserweiterung, die gerade darin ihre praktische Wirksamkeit entfalten soll, dass sie als nicht nur aus praktischen Gründen gesetzte Erkenntniserweiterung gesetzt wird - genau die Trennung zwischen theoretischer Trennung und praktischer Einheit selbst als im praktischen Interesse stehend reflektiert werden muss, i.e. also als eine praktische, unmittelbar widersprüchlich und vollkommen unbestimmt erscheinende Einheit der Einheit und Trennung von Theorie und Praxis.

5. Was also tun angesichts einer solchen potentiell schlechtunendlichen Dialektik? - Eine Antwort auf diese Frage hängt m.E. wesentlich davon ab, ob man sich dafür oder dagegen entscheidet, zu konstatieren, dass dieses gesamte dialektische Problem selbst ein allein im Kontext der praktischen Philosophie auftretendes Problem ist, das es lediglich verlangt, praktisch gelöst werden zu können, ohne dass damit diese Dialektik in die Fundamente dieses Systems selbst hineingetragen werden muss. Nämlich hineingetragen werden in dem Sinne, dass damit eine in sich dialektische Einheit des theoretischen und praktischen Gebrauchs der einen Vernunft zu ihrem eigenen Wesen würde.

Letzteres will Kants Lehre vom Primat des Praktischen nun ausdrücklich nicht, denn diese besteht ja nicht darin, eine prinzipielle Wesensbestimmung der Vernunft daraus zu ziehen, dass diese generell im Sinne einer sich der Theorie bedienenden Praxis bestimmt 
wird $^{56}$, sondern vielmehr soll mit ihr ja nur der Fall eines Widerstreits zwischen spekulativem und praktischem Vernunftgebrauch im Sinne der Gerichtshofmetapher geregelt werden. ${ }^{57}$ Ist in diesem Sinne aber auch die in 3.2 skizzierte Dialektik nur ein sich für die Praxis stellendes Problem, das weder in konstitutiver Weise innerhalb der Kritik des theoretischen Vernunftgebrauchs zur Debatte steht, noch über den Gerichtshofgedanken hinaus eine systemarchitektonische Auswirkung in spekulativer Rücksicht haben muss, dann ist zu fragen, inwiefern nicht auch hier wieder die Lösung in Anschlag gebracht werden kann, dass eine theoretische Problematik dem praktischen Interesse untergeordnet wird, sodass in diesem Sinne geglaubt werden darf.

\subsection{Die Struktur der moralischen Gewissheit in Kants Vernunftglauben}

Als moralische Gewissheit über die Gültigkeit bestimmter theoretischer Sätze wäre Kants praktischer Glaube damit genau diejenige Haltung, in der sich die unter 2. entwickelte Verhältnisbestimmung von theoretischem und praktischem Vernunftgebrauch praktisch motiviert darin Wirklichkeit verleiht, dass sie allem theoretischen Zweifeln an sich selbst, auch und gerade im Sinne der oben skizzierten Dialektik, darin begegnet, dass sie einzig auf ihre praktische Intention abzielend sich schlicht nicht um die oben geschilderte Dialektik sorgt, indem sie ein praktisch motiviertes Zweifeln an allen theoretischen Zweifeln selbst als eine bestimmte Form von praktischer Realität begreift. Dies ganz im Sinne der Aussage, mit der der Abschnitt „Vom Fürwahrhalten aus einem Bedürfnisse der reinen Vernunft“ der zweiten Kritik endet: „Also ist dieses ein Bedürfniß in schlechterdings nothwendiger Absicht und rechtfertigt seine Voraussetzung nicht blos als erlaubte Hypothese, sondern als Postulat in praktischer Absicht; und zugestanden, daß das reine moralische Gesetz jedermann als Gebot (nicht als Klugheitsregel) unnachlaßlich verbinde, darf der Rechtschaffene wohl sagen: ich will, daß ein Gott, daß mein Dasein in dieser Welt auch außer der Naturverknüpfung noch ein Dasein in einer reinen Verstandeswelt, endlich auch daß meine Dauer endlos sei, ich beharre darauf und lasse mir diesen Glauben nicht nehmen; denn dieses ist das einzige, wo mein Interesse, weil ich von demselben nichts nachlassen darf, mein Urtheil unvermeidlich bestimmt, ohne auf Vernünfteleien zu achten, so wenig ich auch darauf $\mathrm{zu}$ antworten oder ihnen scheinbarere entgegen $\mathrm{zu}$ stellen im Stande sein möchte. “58

${ }^{56}$ Vgl. hierzu dann Johann Gottlieb Fichte, Die Bestimmung des Menschen, in: J. G. Fichte-Gesamtausgabe der Bayerischen Akademie der Wissenschaften, Stuttgart - Bad Cannstatt 1962ff., Bd. I 6, S. 265: „Wir handeln nicht, weil wir erkennen, sondern wir erkennen, weil wir zu handeln bestimmt sind; die praktische Vernunft ist die Wurzel aller Vernunft."

${ }^{57}$ Vgl. AA Bd. V, S. 121, Kant betont hier, dass von einer Unterordnung die Rede ist, die nur dann zum Tragen kommt, wenn eine Verbindung des spekulativen mit dem praktischen Vernunftgebrauch „notwendig“ ist. Notwendig nämlich im Sinne der oben ventilierten praktischen Notwendigkeit der Postulate.

${ }^{58}$ AA Bd. V, S. 143. 
1. Die eigentlich interessante Frage scheint mir nun aber zu sein, was für einen Selbstbezüglichkeitsbegriff Kants moralische Gewissheit vor dem Hintergrund des Entwickelten eigentlich benennt? Meinen Versuch einer Antwort auf diese Frage möchte ich nun noch andeuten.

2. Nach dem zuletzt Gesagten, kann offenbar zur gesamten Postulatenlehre selbst eine praktische und eine davon unterschiedene theoretisch reflektierende Haltung eingenommen werden. Damit aber läßt sich in Kants Postulatenlehre im Blick auf die in 3.2 entwickelte Dialektik offensichtlich sehr wohl in klarer Weise eine Differenz zwischen theoretischem und praktischem Gebrauch der Vernunft aufrecht erhalten, sodass damit folgender Aussage Richard Kroners entgegengewirkt werden kann: „Der schwerste Einwurf aber, der sich gegen die ganze Konstruktion des Vernunftglaubens erheben läßt, ist der, daß in ihm theoretische und praktische Vernunft sich nicht zu einer klaren Einheit verbinden, sondern in trüber Weise vermischen und gegenseitig vertilgen. "59 Denn eine Entgegnung auf diesen Vorwurf scheint schlicht darin bestehen zu können, dass auf folgenden Unterschied aufmerksam gemacht wird: - Es ist das Eine, wenn die in 3.2 entfaltete Dialektik im Sinne der mit 3.3 und 3.4 vorgeschlagenen Lösung allein mit praktischen Mitteln aufgehoben wird. D.h. also durch eine Regelung, die allein sagt, wer den Primat führt, ohne dass dabei der Versuch unternommen wird, den theoretischen Zweifel selbst mit einem theoretischen Argument zu beseitigen. - Ein Anderes ist es, wenn diese Dialektik im Sinne von 3.2 voll zur Geltung kommen darf.

3. Die Tatsache, dass die in 3.2 entfaltete Dialektik in Einem in theoretischer Rücksicht als Folge des Primats des Praktischen verstanden werden kann und in praktischer Rücksicht als Hindernis für denselben, scheint mir nun aber noch einmal auf einen weiteren, entscheidenden Punkt hinzudeuten. Nämlich darauf, dass im in 3.3 und 3.4 bereits angedeuteten Sinne auch der Begriff des Primats des Praktischen als praktischer Begriff möglicherweise gerade eine willentlich gesetzte Unbegreiflichkeit einschließen muss.

4. Sollte dies richtig sein, würde sich damit ganz im Sinne der Schlussanmerkung zur Grundlegung zur Metaphysik der Sitten ${ }^{60}$ auch hinsichtlich Kants Lehre vom Primat des Praktischen die methodisch-grundlegende Überlegung aufdrängen, dass gerade die theoretisch restringierte Thematisierung des Primats des Praktischen als die praktisch bestimmte Thematisierung des Primats des Praktischen erscheinen muss.

5. Genau diese Überlegung wäre dann aber auch noch einmal ein wichtiger Punkt in der Frage nach dem Kants moralischer Gewissheit zugrundeliegenden Selbstbezüglichkeitsbegriff.

\footnotetext{
${ }^{59}$ Vgl. Richard Kroner, Von Kant bis Hegel, 2. Aufl. Tübingen 1961, S. 206.
}

${ }^{60} \mathrm{Vgl}$. AA Bd. IV, S. 463: „Es ist also kein Tadel für unsere Deduction des obersten Princips der Moralität, sondern ein Vorwurf, den man der menschlichen Vernunft überhaupt machen müßte, daß sie ein unbedingtes praktisches Gesetz (dergleichen der kategorische Imperativ sein muß) seiner absoluten Nothwendigkeit nach nicht begreiflich machen kann; denn daß sie dieses nicht durch eine Bedingung, nämlich vermittelst irgend eines zum Grunde gelegten Interesse, thun will, kann ihr nicht verdacht werden, weil es alsdann kein moralisches, d.i. oberstes Gesetz der Freiheit sein würde. Und so begreifen wir zwar nicht die praktische unbedingte Nothwendigkeit des moralischen Imperativs, wir begreifen aber doch seine Unbegreiflichkeit, welches alles ist, was billigermaßen von einer Philosophie, die bis zur Grenze der menschlichen Vernunft in Principien strebt, gefordert werden kann." 
Denn nach dem im Vorausgegangenen Entwickelten scheint zu gelten, dass die moralische Gewissheit den Glauben an und in sich selbst, nämlich ein An-und-Fürsichsein von Praxis zu sein, nicht zuletzt gerade darin gesichert weiß, dass sie begreift, dass sie alle theoretischen Zweifel und Vernünfteleien gegen sich im Sinne des in ihr wirklichen praktischen Interesses ${ }^{61}$ im Sinne eines praktisch-bestimmt verstandenen Primats des Praktischen von sich halten kann, darf und muss.

Vielleicht könnte man sogar soweit gehen, zu sagen, dass die Vollstruktur der moralischen Gewissheit nur zu begreifen ist, wenn sie als konkreter Ausdruck dieser zuletzt unter Verweis auf das Ende der Grundlegung angerissenen, die Architektonik des Kantschen Systems so grundlegend bestimmenden Idee eines praktischen Begriffs von Selbstbezüglichkeit verstanden wird. Denn in der Tat scheint zu gelten, dass ihre „am Ort der Praxis“ angestellten Reflexionen ihren „Ort“ und Vergewisserungspunkt letztlich gerade in diesem Selbstbezüglichkeitsbegriff zu finden haben. Denn „am Ort der Praxis zu denken“, heißt innerhalb der Postulatenlehre im Sinne des von ihr propagierten „moralisch conesquenten Denkens“62 m.E. in letzter Konsequenz kurzum, genau diesen Begriff praktischer Selbstbezüglichkeit denken zu können und denken zu wollen.

\subsection{Rückblick und Ausblick. Die Postulatenlehre auf dem Weg zum Absoluten ${ }^{63}$}

1. Gezeigt werden sollte in den vorausgegangenen Überlegungen vor allem, dass es das Eine ist, in Kants innerhalb seiner Lehre eines praktischen Vernunftglaubens thematisierten Struktur moralischer Gewissheit Selbstbezüglichkeitsprobleme auszumachen, um dann deren dialektische Weiterentfaltung zu verfolgen. - Etwas Anderes ist es, den Preis zu antizipieren, den dies haben könnte, nämlich den Primat des Praktischen in eine Dialektik zu überführen, die den zuletzt benannten praktischen Begriff davon, Selbstbezüglichkeitsprobleme zu thematisieren und zu behandeln, gerade aufgibt, - nämlich einen praktischen Begriff von Selbstbezüglichkeit, der interessanterweise gerade darin bestimmt zu sein scheint, inwieweit in einem unter dem Primat des Praktischen stehenden System Selbstbezüglichkeit theoretisch-dialektisch thematisiert wird und inwieweit gerade nicht.

2. Nicht ausgeschlossen zu sein scheint damit aber gerade die Möglichkeit des Versuchs, auch diesem Sachverhalt noch eine ebensosehr praktische wie theoretische Wendung zu geben - sei es in einer noch direkt an Kants dritter Kritik orientierten Vereinigung von Bewusstem und Bewusstlosem in einer Philosophie der Geschichte, Natur und Kunst ${ }^{64}$, oder sei es auch in einer dialektischen, mit dem System ihres Erscheinens in besonderer Bestimmtheit identisch konstruierten Idee $^{65}$-, um gerade auch hiermit vielleicht noch den

${ }^{61} \mathrm{Vgl}$. abermals AA Bd. V, S. 143.

${ }^{62} \mathrm{Vgl}$. AA Bd. V, S. 451 Anm.

${ }^{63}$ Vgl. hierzu auch „Die Moraltheologie auf dem Weg zum Absoluten“, in: Dieter Henrich, Grundlegung aus dem Ich, Frankfurt a. M. 2004, SS. 1467-1548.

${ }^{64}$ Vgl. F.W.J. Schelling, System des transzendentalen Idealismus, hrsg. von H. Brandt und P. Müller, Hamburg 1992 , insbes. S. $283 \mathrm{ff}$.

${ }^{65}$ Vgl. G.W.F. Hegel, Enzyklopädie der philosophischen Wissenschaften im Grundrisse, \$ 15, GW Bd. 20, S. 56. 
Versuch einer Weiterentfaltung dessen zu unternehmen, was das sogenannte Älteste Systemprogramm des Deutschen Idealismus auf der Schwelle zu den großen nachkantischen Systemen der klassischen deutschen Philosophie selbst als diese Schwelle ausspricht, nämlich dies: „Da die ganze Metaphysik künftig in die Moral fällt - wovon Kant mit seinen beiden praktischen Postulaten nur ein Beispiel gegeben, nichts erschöpft hat -, so wird diese Ethik nichts anderes als ein vollständiges System aller Ideen oder, was dasselbe ist, aller praktischen Postulate sein." 66

${ }^{66}$ Vgl. Das älteste Systemprogramm des deutschen Idealismus, zitiert nach G.W.F. Hegel, Werke in 20 Bänden, neu hrsg. auf Grundlage der von 1832-45 „durch einen Verein von Freunden des Verewigten“ edierten Werke unter Redaktion von Eva Moldenhauer und Karl Markus Michel, Frankfurt a. M. 1986, Bd. 1, S. 234. 